\title{
Stellar Populations in the Local Group of Galaxies
}

\author{
Eva K. Grebel \\ Astronomical Institute of the University of Basel, Venusstrasse 7, CH-4102 Binningen, Switzerland
}

\begin{abstract}
The characteristics and properties of the stellar populations and evolutionary histories of Local Group galaxies are summarized and compared to predictions of cosmological models. No clear signature of the re-ionization epoch is observed; in particular, there is no cessation of star formation activity in low-mass dwarf galaxies at the end of re-ionization. Arguments against the morphological transformation of dwarf irregular into dwarf spheroidal galaxies are derived from their pronounced evolutionary differences at early epochs as evidenced by the offset in the metallicityluminosity relation between gas-rich and gas-poor dwarfs. While there is increasing evidence for past and ongoing accretion events the overall importance of dwarf galaxies as building blocks remains unclear considering their differences in modes of star formation and detailed chemistry.
\end{abstract}

Keywords: Dwarf galaxies (elliptical, irregular, and spheroidal) - Magellanic Clouds and other irregular galaxies - Origin, formation, evolution, age, and star formation - Chemical composition and chemical evolution - Stellar content and populations; radii; morphology and overall structure - Galactic halos

PACS: 98.56.Wm, 98.56.Si, 98.62.Ai, 98.62.Bj, 98.62.Lv, 98.62.Gq

\section{THE LOCAL GROUP}

The Local Group of galaxies is dominated by two massive spirals, M31 and the Milky Way. The Local Group (LG) is our immediate cosmic neighborhood, a sparse galaxy group with a zero-velocity radius of $1 \mathrm{Mpc}$ (Karachentsev et al. 2002a). Two dominant galaxies also characterize a number of other nearby galaxy groups, and zero-velocity radii of 1 to $1.2 \mathrm{Mpc}$ are typical for these poor groups. Thirty-eight galaxies are currently known to be located within the LG's radius, but the galaxy census of the LG is still incomplete. While all of its luminous members have been detected, new faint member candidates with very low surface brightnesses are still being discovered (e.g., Zucker et al. 2004a). In spite of its small size and its small number of galaxies the LG plays a prominent role in astrophysical research. For reviews of the LG, see Grebel $(1997,1999$, 2000, 2001), Mateo (1998), and van den Bergh (1999, 2000). In the present contribution, I will mainly concentrate on the dwarf galaxies of the LG.

The proximity of the galaxies of the LG permits us to study them at the highest possible resolution, enabling us to resolve these galaxies into individual stars down to very faint magnitudes. For example, with the Hubble Space Telescope, point sources down to an apparent magnitude of $\approx 30$ have been detected in M31 (Brown et al. 2003), corresponding to the magnitudes of stars well below the Population II main-sequence turn-off at that distance. To date, the LG is the only location where we can observe the lowest stellar masses, measure the oldest stellar ages, determine metallicities and element abundance ratios of individual stars of high ages, and measure detailed stellar 
kinematics. In other words, the LG is unique in affording the highest level of detail and accuracy for stellar population studies. Much of this research has only become possible in recent years thanks to the superior angular resolution and sensitivity of the Hubble Space Telescope (HST), and thanks to the advent of powerful ground-based optical/near infrared 8 to $10 \mathrm{~m}$ class telescopes, which provide sufficient sensitivity for medium and high-resolution stellar spectroscopy of individual stars in nearby galaxies. It is now becoming possible to uncover the evolutionary histories of these galaxies in unprecedented detail.

Furthermore, the LG contains a variety of different galaxy types. While massive earlytype galaxies are absent, the LG still provides us with galaxies covering five orders of magnitude in galaxy masses, a variety of different morphological types, very different star formation histories, and a range of different dominant ages and metallicities. Within the small volume of the LG, galaxies occur in environments ranging from immediate proximity to massive galaxies to fairly isolated locations without nearby neighbors. Hence we can expect to eventually obtain first-hand measurements of the impact of environment on galaxy evolution, at least on small scales.

All in all, by studying the stellar populations of galaxies in the LG and by combining them with detailed knowledge of the other components of these galaxies such as gas, dust, and non-luminous matter we can learn about galaxy properties at the highest possible level of detail. The LG permits us to study stellar evolution at a range of different metallicities and ages not afforded in that combination in the Milky Way; e.g., the evolution of young, massive stars at low metallicities. Moreover, the LG permits us to carry out tests of cosmological galaxy evolution theories, for instance with respect to time scales and number and magnitude of accretion events. Hence here we can conduct "near-field cosmology" (see Freeman \& Bland-Hawthorn 2002). The LG is particularly well-suited to learn more about the formation and evolution of disk galaxies, whose theoretical foundations are still poorly understood. Understanding all of these processes in an environment where we can learn about them in detail is the precondition for understanding distant, unresolved galaxies.

\subsection{The galaxy content of the Local Group}

We currently know of 38 galaxies within the zero-velocity surface of the Local Group. Whether the outermost galaxies are indeed gravitationally bound to the LG remains unclear. Indeed, reliable orbits are not yet known for any of the LG galaxies, and proper motion measurements exist only for the closest Milky Way companions. The uncertainties of these measurements are still large, but this situation should change once data from the planned astrometric satellite missions - Gaia (an ESA mission that will scan repeatedly the entire sky) and the Space Interferometry Mission (SIM, a NASA mission that will perform pointed observations with even higher accuracy) - become available. These missions will probably not start before 2011 .

As mentioned before, the LG galaxy census is still incomplete. Apart from missing orbital information, galaxies of very faint surface brightness are still being detected as sky surveys become more sensitive or as data mining techniques are being improved. 
During the past six years, this led to the discovery of one fairly isolated new dwarf spheroidal (dSph) galaxy in the Local Group (Cetus; Whiting, Irwin, \& Hau 1999), and to the discovery of four new dSph companions of M31 (Armandroff, Davies, \& Jacoby 1998; Armandroff, Jacoby, \& Davies 1999; Karachentsev \& Karachentseva 1999, Grebel \& Guhathakurta 1999, Zucker et al. 2004a, Harbeck et al. 2005). The conclusion of data mining existing photographic all-sky surveys (Karachentsev et al. 2000; Whiting, Hau, \& Irwin 2002) makes the existence of additional faint, Draco- or Ursa-Minor-like $\mathrm{dSphs}$ seem unlikely unless they are hidden behind regions of high Galactic extinction, particularly in the zone of avoidance. On the other hand, the discovery of And IX (Zucker et al. 2004a) with an unusually low surface brightness of only $\mu_{V, 0} \sim 26.8 \mathrm{mag} \mathrm{arcsec}^{-2}$ indicates that additional very sparse, very faint dwarfs may yet have to be discovered. At present, though, there is little reason to believe that there may still be hundreds of yet to be detected faint objects present in the LG.

Very faint, sparse, low-surface-brightness objects like And IX, which appear to be dominated by ancient, metal-poor stars (Harbeck et al. 2005) are of immense interest from the point of view of galaxy evolution. Do they show signatures of cosmic re-ionization squelching? Do they contain the purest, most metal-poor, most ancient populations known? Or do they show evidence for population gradients in their old population as were found in a number of other ancient dSphs (Harbeck et al. 2001)? Do these objects show large metallicity spreads as measured in other seemingly purely old dSphs (Shetrone, Côte, \& Sargent 2001), which in turn indicate extended episodes of star formation (Ikuta \& Arimoto 2002)? How did these objects survive? Are they entirely dominated by dark matter? Are they regular dSph galaxies that simply are fainter and sparser than the previously known members of this galaxy class? Yet from a cosmological point of view, objects of this type may appear to be of little interest since they may not significantly contribute toward solving the "missing satellite problem" (Klypin et al. 1999; Moore et al. 1999) unless detected in vast numbers.

Not only low surface brightness, scarcity of stars, high foreground extinction, and unknown orbits for distant LG member candidates contribute to an incomplete LG galaxy census, but also accretion events. We still know little about the actual number of accretion events, about the times when these events occurred, and about the nature of the accreted galaxies (see also the Section 1.4 and the contribution of Majewski in these proceedings). The number of 38 probable LG members comprises all galaxies listed in Grebel, Gallagher, \& Harbeck (2003) including the Sagittarius dwarf galaxy, which is currently being accreted by the Milky Way. In addition, it includes the giant stream around M31 (Ibata et al. 2001), which is very likely the remnant of a disrupted galaxy, and the newly discovered M31 satellite And IX (Zucker et al. 2004a). Not included are other possible streams and accretion remnants such as the Monoceros feature (Newberg et al. 2002) and Canis Major (Martin et al. 2004a) since their nature as dwarf galaxy remnants remains disputed (see Section 1.4). Depending on the nature of these and other stellar overdensities and kinematically identified features in the Milky Way and in M31, the LG census may need to be increased by at least up to six or more galaxies if one wishes to account for still measurable accretion events. If these features are confirmed as minor mergers, then it becomes a matter of definition whether their progenitors should be included in the current LG member census. 


\subsection{Galaxy types in the Local Group}

Approximately $90 \%$ of the LG's luminosity is contributed by its three spiral galaxies, but the vast majority of the LG galaxy population are satellite and dwarf galaxies. The boundaries between larger galaxies and dwarf galaxies are poorly defined. We adopt here the usual convention of calling every galaxy with an absolute magnitude of $M_{V}>-18$ mag a dwarf galaxy. This simple magnitude criterion then leaves us with 34 dwarf galaxies (though admittedly the absolute magnitude of the progenitor of M31's "great stream" is yet unknown, and that of the Sagittarius dSph is poorly constrained). Apart from the three spirals, only the Large Magellanic Cloud is more luminous than the above luminosity threshold. Yet M32 is often not considered a dwarf galaxy despite its lower luminosity since it exhibits similar structural properties as giant ellipticals.

Most of the LG galaxies belong to one of four basic classes: Spirals, dwarf irregular galaxies, dwarf elliptical galaxies, and dSphs. In the following paragraphs the main characteristics of the dwarf galaxy classes are summarized following Grebel (2001):

Dwarf irregular galaxies (dIrrs) are gas-rich galaxies with an irregular optical appearance usually dominated by scattered H II regions. They typically have V-band surface brightnesses of $\mu_{V} \lesssim 23 \mathrm{mag} \operatorname{arcsec}^{-2}$, H I masses of $M_{\mathrm{HI}} \lesssim 10^{9} \mathrm{M}_{\odot}$, and total masses of $M_{\text {tot }} \lesssim 10^{10} \mathrm{M}_{\odot}$. The stellar populations of dIrrs range from ancient stars with ages $>10$ Gyr to ongoing star formation. Star formation appears to have proceeded largely continuously, although amplitude variations in the intensity or rate of star formation may reach a factor of three. The $\mathrm{H}$ I distribution is usually clumpy and more extended than even the oldest stellar populations. When considering only the distribution of older stellar populations (i.e., of red giants and red clump stars, which trace populations older than $\sim 1$ Gyr), irregulars and dIrrs exhibit a highly regular distribution shaped like an (elongated) disk (LMC; van der Marel 2001) or like a spheroid (example: Small Magellanic Cloud (SMC); Zaritsky et al. 2000). In low-mass dIrrs gas and stars may exhibit distinct spatial distributions and different kinematic properties. Metallicities tend to increase with decreasing age in the more massive dIrrs, indicative of enrichment and of an age-metallicity relation (see also Fig. 3 in Grebel 2004). For low-mass dIrrs detailed measurements are still lacking. Solid body rotation is common among the more massive dIrrs, whereas low-mass dIrrs seem to be dominated by random motions without evidence of rotation. DIrrs are found in galaxy clusters, groups and in the field.

Dwarf elliptical galaxies (dEs) are spherical or elliptical in appearance, tend to be found near massive galaxies (in the Local Group they are all companions of M31), usually have little or no detectable gas, and tend not to be rotationally supported. Note that examples of rotating dEs beyond the Local Group have been found (e.g., Pedraz et al. 2002). DEs are compact galaxies with high central stellar densities. They are typically fainter than $\mathrm{M}_{V}=-17 \mathrm{mag}$, have $\mu_{V} \lesssim 21 \mathrm{mag} \operatorname{arcsec}^{-2}, M_{\mathrm{HI}} \lesssim 10^{8} \mathrm{M}_{\odot}$, and $M_{\text {tot }} \lesssim 10^{9}$ $\mathrm{M}_{\odot}$. DEs may contain conspicuous nuclei (nucleated $\mathrm{dEs}$ or $\mathrm{dE}, \mathrm{N}$ ) that may contribute up to $20 \%$ of the galaxy's light. The fraction of dE,N is higher among the more luminous dEs. An example for a non-nucleated dE in the Local Group is NGC 185, whereas

NGC 205 is a dE,N. Sérsic's (1968) generalization of a de Vaucouleurs $r^{1 / 4}$ law and exponential profiles describe the surface density profiles of nucleated and non-nucleated dEs and dSphs best (Jerjen et al. 2000). DEs typically contain old and intermediate-age 
populations (i.e., populations older than $10 \mathrm{Gyr}$ and populations in an age range of $\sim 2$ to $10 \mathrm{Gyr}$ ), but the fractions of these populations vary, and even present-day star formation may be observed.

Dwarf spheroidal galaxies (dSphs) are diffuse, gas-deficient, low-surface-brightness dwarfs with very little central concentration. They are not always distinguished from dEs in the literature. DSphs are characterized by $\mathrm{M}_{V} \gtrsim-14 \mathrm{mag}, \mu_{V} \gtrsim 22 \mathrm{mag} \operatorname{arcsec}^{-2}$, $M_{\mathrm{HI}} \lesssim 10^{5} \mathrm{M}_{\odot}$, and $M_{t o t} \sim 10^{7} \mathrm{M}_{\odot}$. They include the optically faintest galaxies known. Their stellar populations tend to be either almost purely old or a mix of old and intermediate-age populations. The luminosity functions (and by inference the mass functions) of dSphs have been found to be "normal" and in excellent agreement with those of Galactic globular clusters (Grillmair et al. 1998; Wyse et al. 2002). DSphs are usually found in close proximity of massive galaxies (counterexamples in the LG: Cetus, Tucana) and are generally not supported by rotation. Since most published measurements concentrated on the central regions of dSphs, the possibility of slowly rotating $\mathrm{dSphs}$ cannot be excluded. The central velocity dispersions of dSphs indicate the presence of a significant dark component when virial equilibrium is assumed. However, not all dSphs are in virial equilibrium (e.g., Ursa Minor shows indications of being tidally distorted by the Milky Way; for instance, Palma et al. 2003). Interestingly, the radial velocity dispersion profiles of dSphs show a marked drop at large radii (Wilkinson et al. 2004). Its interpretation and the apparent existence of a kinematically cold stellar population at the outermost radii is not yet understood. The metallicity-luminosity relations of dSphs and dIrrs show the usual trend of increasing metallicity with increasing galaxy luminosity, but the relations are offset from each other: DSphs have higher mean stellar metallicities at a given optical luminosity (Grebel et al. 2003 and Section 3.1).

\subsection{Morphological segregation}

As already indicated, the distribution of the different types of galaxies within the LG is not random, but is determined by the location of the two most massive spiral galaxies in the LG, M31 and the Milky Way. A sketch of the three-dimensional distribution of galaxies within the LG is shown in Grebel (1999; in Fig. 3). In Fig. 1 the distribution of gas-poor, low-mass dwarfs and of gas-rich, higher-mass dwarfs is shown. The gas-poor dwarfs - primarily dSphs - are strongly clustered around the closest massive spiral, whereas the gas-rich dwarfs (dIrrs) exhibit less of a tendency toward close concentration around massive galaxies and are more widely distributed. The differing distribution of different morphological galaxy types is also known as morphological segregation and is observed in nearby galaxy groups and galaxy clusters as well.

Similarly, the H I mass of dwarf galaxies tends to increase with increasing distance to a massive primary (Grebel et al. 2003; their Fig. 3). These trends indicate that environment may have a significant impact on the evolution of low-mass galaxies. Indeed, it is tempting to speculate that these trends are the result of morphological transformations due to the influence of massive galaxies, e.g., via tidal or ram pressure stripping (see Mayer et al. 2001 for simulations). One vital ingredient to verify this hypothesis is once again information about the orbits of the companion galaxies. 


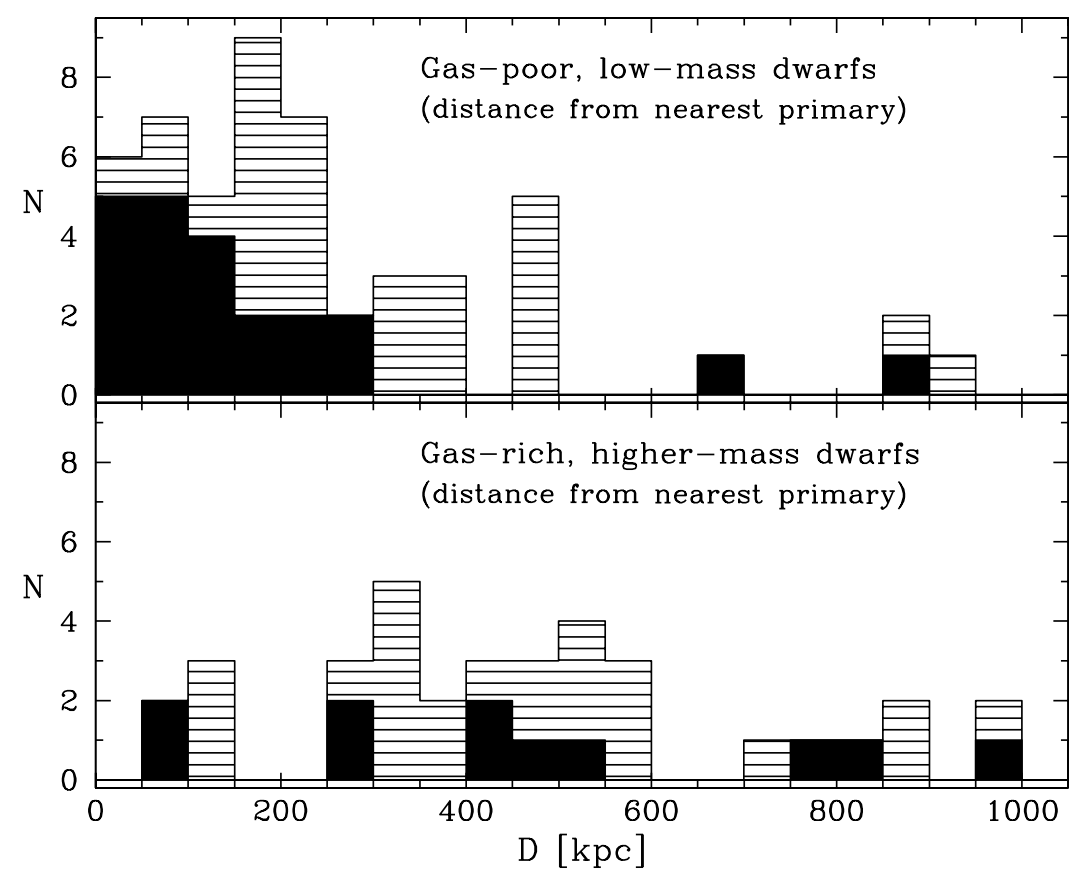

FIGURE 1. Morphological segregation in the number distribution $\mathrm{N}$ of different types of galaxies in the Local Group (solid histograms) and in the M81 and Centaurus A groups (hashed histograms) as a function of distance D to the closest massive primary (updated version of Fig. 1 in Grebel 2004).

\subsection{Direct evidence for harassment and accretion}

If accretion is the primary mechanism for the growth and evolution of massive galaxies as predicted by cosmological models, then we should be able to find evidence for these processes in our immediate neighborhood. (1) The study of the structural properties of nearby galaxies can reveal whether external tidal forces are distorting them. (2) The detection of extratidal stars and streams around and within massive galaxies is evidence for ongoing harassment and accretion events. (3) The stellar content, population properties, and chemistry of nearby galaxies allow us to constrain to what extent these kinds of objects could have contributed as building blocks to more massive galaxies.

The clearest evidence for ongoing accretion are the extended tidal stream of the Sagittarius dSph galaxy (Ibata, Gilmore, \& Irwin 1994), which has now been traced around the entire Milky Way using M giants identified in the Two Micron All-Sky Survey (2MASS) (Majewski et al. 2003), and the giant stream of metal-rich giants in the halo of M31 (Ibata et al. 2001; Ferguson et al. 2002). Additional stellar overdensities have been detected in the Milky Way using various photometric data sets including 2MASS and the Sloan Digital Sky Survey (SDSS): The Monoceros feature (Newberg et al. 2002; Yanny et al. 2003), which may be a tidal tail connected with the Canis Major overdensity (Martin et al. 2004a). The interpretation of Canis Major is disputed; suggestions include that it is part of the Galactic warp or flare (Momany et al. 2004) or indeed the center of another possibly disrupted dSph within the Milky Way (Martin et al. 2004a, 2004b). Additional Galactic stellar overdensities have been identified (for instance, Triangulum- 
Andromeda; Rocha-Pinto et al. 2004, which may be part of the tidal tail of a more distant disrupted dwarf). Other suggestions of evidence of dwarf galaxy accretion are based on the identification of moving groups and radial velocity surveys (e.g., Gilmore, Wyse, \& Norris 2002). However, not only disrupted dwarf galaxies, but even globular clusters in advanced stages of accretion may produce extended stellar tidal tails (Odenkirchen et al. 2001; 2003). These tidal features are valuable also as tracers of the Galactic potential. The most luminous and most massive Galactic globular cluster, $\omega$ Centauri, contains a range of different ages and a large metallicity spread. A popular explanation for its unusual properties is that $\omega$ Cen may be the core of an accreted dwarf galaxy (see van Leeuwen, Hughes, \& Piotto 2002 for details). - Possible additional tidal features in and near M31 have been reported by Morrison et al. (2003) and Zucker et al. (2004b).

The Magellanic Clouds and the Milky Way are interacting with each other as evidenced by, e.g., the gaseous Magellanic Stream and the Magellanic Bridge (e.g., Brüns et al. 2005), although the interpretation of these features as being primarily due to tidal (e.g., Putman et al. 1998) or ram pressure effects (Mastropietro et al. 2005) remains controversial. The twisted isophotes of the M31 companions M32 and NGC 205 may be caused by tidal interaction with M31 (Choir, Guhathakurta, \& Johnston 2002). The nearby Galactic dSph satellite Ursa Minor shows a distorted, S-shaped surface density profile possibly caused by tidal interaction with the Milky Way (Palma et al. 2003). On the other hand, the drop in the velocity dispersion profiles of Draco and Ursa Minor at large radii (Wilkinson et al. 2004) and the lack of a large depth extent of Draco (Klessen, Grebel, \& Harbeck 2003) would seem to argue against ongoing tidal disruption.

\section{THE EARLIEST EPOCH OF STAR FORMATION}

According to hierarchical structure formation scenarios, low-mass systems should have been the first sites of star formation in the Universe. The first star formation events may have occurred as early as at a redshift of $\mathrm{z} \sim 30$, an epoch unobservable for us with our present tools. Larger systems should then have formed through hierarchical merging of smaller systems, leading to the idea of dwarf galaxies as building blocks of more massive galaxies. Hence one important test to carry out is to compare the properties of the old populations in dwarf galaxies with those in massive galaxies to investigate how similar or dissimilar they are. If today's dwarf galaxies are the few survivors of a once much more numerous, since accreted low-mass galaxy population, and if dwarf galaxy accretion is the primary process governing the formation of more massive galaxies, then detailed studies of their old stellar populations should reveal very similar properties.

A number of cosmological models predict that cosmic re-ionization will squelch star formation in low-mass substructures, and that galaxies less massive than $10^{8}$ to $10^{9}$ $\mathrm{M}_{\odot}$ will lose their star-forming material through photoevaporation during re-ionization (e.g., Ferraro \& Tolstoy 2000; Dekel \& Woo 2003; Susa \& Umemura 2004). As a consequence, low-mass galaxies must form their stars prior to re-ionization (e.g., Tassis et al. 2003) and need to contain ancient populations. Furthermore, one would expect a sharp drop and indeed a complete cessation of star-forming activity after re-ionization is complete. A third consequence is that the oldest populations in high-mass galaxies must be either as old as those in low-mass galaxies, or younger. 


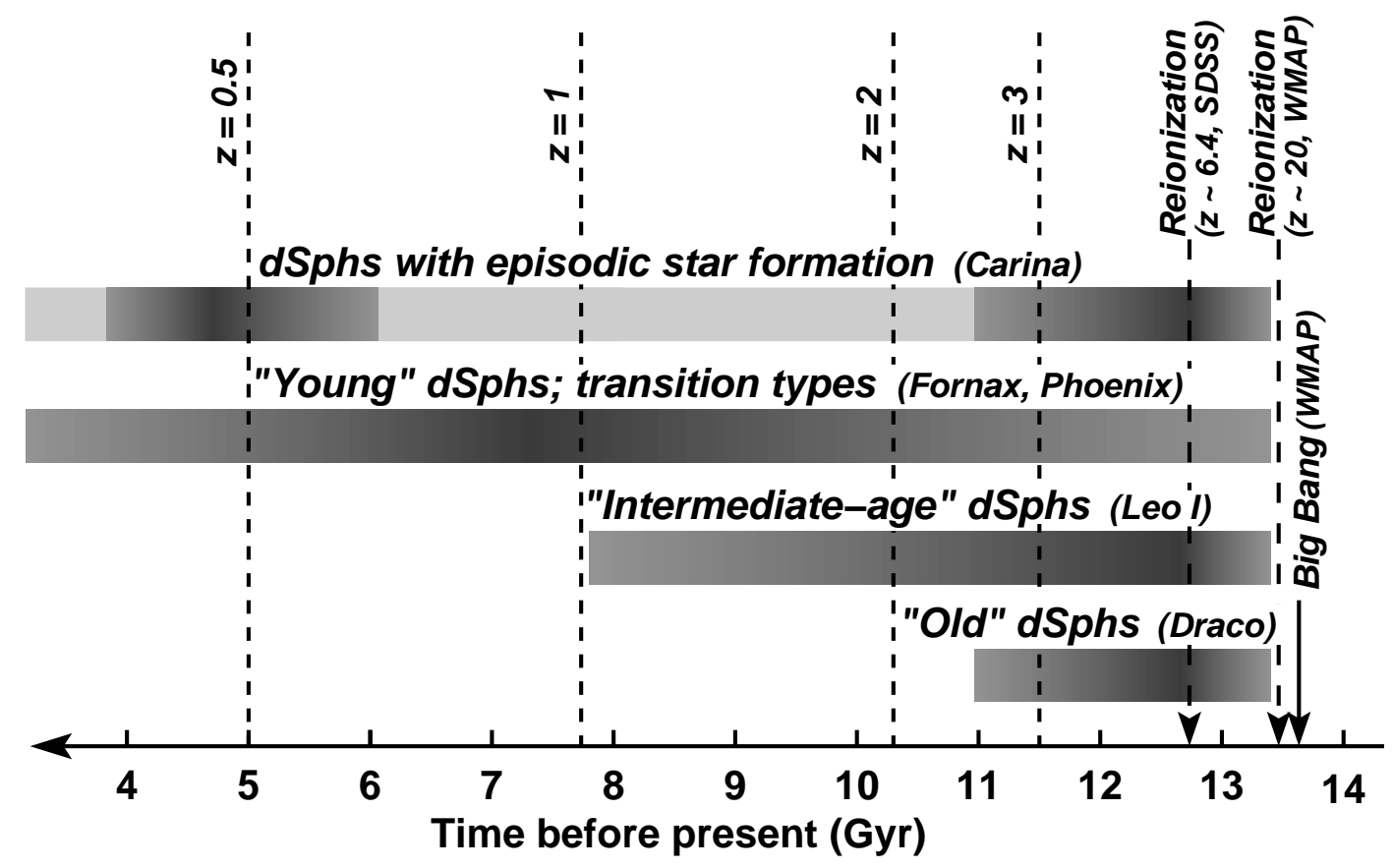

FIGURE 2. Bar diagram indicating the approximate duration of star formation episodes in low-mass galaxies $\left(\sim 10^{7} \mathrm{M}_{\odot}\right)$. The approximate beginning and end of the re-ionization epoch are indicated (based on results from WMAP and from the Sloan Digital Sky Survey). The predicted cessation of star formation in low-mass galaxies, esp. in dSphs, is not observed. For more details, see also Grebel \& Gallagher (2004).

These are testable predictions that can be investigated by exploiting the fossil stellar record in nearby galaxies. The LG is an ideal target since here the oldest populations are resolved and can be accessed with HST and increasingly also with large ground-based telescopes operating with high angular resolution. This requires age dating of old populations. The most accurate ages can be obtained for resolved stellar populations. For old populations the most age-sensitive feature is the old main-sequence turn-off (MSTO), where via differential age-dating techniques internal accuracies of less than $1 \mathrm{Gyr}$ can be obtained. Comparison objects are usually ancient Galactic globular clusters of the same metallicity as the target population. Absolute ages are more difficult to determine since here one needs to rely on isochrones, which makes the resulting ages model-dependent. But also differential techniques have a number of drawbacks: They require very deep, high-quality photometry reaching at least 2 mag below the MSTO, which is a challenge in more distant galaxies. They require stellar populations sufficiently numerous to produce a measurable MSTO, which necessarily limits us to Population II stars (note that to date not a single Population III star candidate has ever been detected beyond the Milky Way). Ideally, one wishes to compare populations with the same $[\alpha / \mathrm{Fe}]$ ratio. (Establishing to what extent this condition is met and what the consequences are will be an important area for large ground-based telescopes in the coming years.) Other effects such as diffusion may further affect relative measurements; see Chaboyer's contribution in these proceedings for details.

For a more detailed discussion of the results of differential age dating as applied 
to ancient Milky Way populations and nearby dwarfs I refer to Grebel (2000) and Grebel \& Gallagher (2004). Here only the results shall be summarized, which are based on Galactic and extragalactic globular clusters and field populations with MSTO photometry: (1) Old populations are ubiquitous, but their fractions vary. There is not a single dwarf galaxy studied in sufficient detail without an old population. (2) There is evidence for a common epoch of star formation. The ancient Population II in the Milky Way, the LMC, and the Galactic dSphs are coeval within $\sim 1$ Gyr (which is consistent with the building block scenario). (3) In contrast to predictions from cold dark matter models, no cessation of star formation activity is observed during or after re-ionization. (4) Instead, even the least massive galaxies known, the dSphs, show evidence for star formation extending over many Gyr. This holds even for those dSphs entirely dominated by very old populations - their metallicity spread requires enrichment due to star formation extending over several Gyr.

It is certainly correct to point out that the accuracy of age determinations does not permit us yet to confidently state when exactly early star formation began and ended in dSphs. Nevertheless, the presence of ancient populations in dSphs is a fact. The large metallicity spread in old dSphs has been proven spectroscopically. Furthermore, the presence of intermediate-age populations in addition to ancient stars in many though not all dSphs cannot be refuted. In only one of these dSphs (Carina) clearly episodic star formation with well-separated "bursts" has been observed (Smecker-Hane et al. 1994); in all others star formation appears to have proceeded fairly continuously. The characteristics of the star formation and enrichment histories of dSphs contradict the cosmological predictions mentioned earlier, particularly the suggestion of complete photoevaporation of baryonic material not yet turned into stars.

There are several possible ways out of this quandary. Either the above quoted cosmological models are wrong, or do not take other effects that might prevent photoevaporation into account, or the galaxies we observe today as dSphs once were substantially more massive. In case if the latter, today's dSphs would once have needed to be at least a factor of 100 more massive. An oversimplified estimate shows that at the times when dSphs were forming stars, they should have had at least 10 times more baryonic mass if we assume a star formation efficiency of $10 \%$. Their present-day baryonic masses are of the order of $10^{6} \mathrm{M}_{\odot}$, so an increase by one order of magnitude would not yet change the overall estimated masses of dSphs drastically, but it would considerably alter their mass-to-light ratios. More detailed calculations and more observational data are needed to investigate whether substantially more massive $\mathrm{dSph}$ progenitors are plausible. The differences in the metallicity-luminosity relation of dSphs and dIrrs seem to rule out dIrrs as progenitors of dSphs (Grebel et al. 2003).

\section{STAR FORMATION HISTORIES AND METALLICITIES}

Much has been said already about star formation histories in the preceding sections. In the past years, considerable effort has been spent on uncovering star formation histories of nearby dwarf galaxies by photometric means, i.e., via color-magnitude diagrams of

the resolved stellar populations of the stars in these galaxies. Basically, very detailed star formation histories are derived photometrically through synthetic color-magnitude 
diagrams (CMDs) generated from isochrones assuming an initial mass function and a variety of different time-dependent star formation and chemical evolution histories. The synthetic CMDs are compared to the observed CMDs using various statistical techniques until the best match is found (e.g., Tosi et al. 1991; Gallart et al. 1996; Tolstoy \& Saha 1996; Dolphin 1997; Holtzman et al. 1997). These methods necessarily have to make a number of assumptions (such as which initial mass function and which binary fraction to adopt), rely strongly on the chosen set of isochrones, and are plagued by the agemetallicity degeneracy, but yield surprisingly similar results in spite of the different approaches of different groups (see contributions in Lejeune \& Fernandes 2002).

The information gained from deep CMDs can be complemented by information provided by individual age tracer stars such as Wolf-Rayet stars, carbon stars, RR Lyrae stars, etc. (see Grebel 1997, 1999) to yield a more complete picture of the star formation histories of the galaxies in question. In particular, such tracer populations are valuable when their specific host populations are too sparse to appear prominently in the CMDs. Furthermore, star formation histories vary as a function of position with the oldest populations being typically the most extended ones (e.g., Harbeck et al. 2001), hence ideally one wishes to cover the entire target galaxy.

Our current knowledge about the modes of star formation in LG dwarf galaxies can be summarized as follows: Irregular and dIrr galaxies are characterized by largely continuous star formation with amplitude variations of factors $2-3$, governed mainly by internal, local processes. In the more massive irregulars with large gas reservoirs, star formation is likely to proceed for another Hubble time (Hunter 1997). Low-mass socalled dIrr/dSph transition-type galaxies are characterized by currently very low star formation rates and may eventually turn into quiescent dSphs. A detailed review of the evolutionary histories of these galaxies, large-scale and small-scale star formation properties, and their chemical evolution is given in Grebel (2004).

$\mathrm{DE}$ and $\mathrm{dSph}$ galaxies also tend to have continuous star formation rates, but with decreasing intensity. Some had their peak activity at very early ages, others several Gyr ago. Star formation tends to be longest-lasting in the centers of these galaxies, and in a number of cases age (and possibly metallicity) gradients are observed (Harbeck et al. 2001). Repetitive or episodic star formation, as mentioned earlier, has so far only found in Carina. For more detailed reviews, see Grebel $(2000,2001)$. A major puzzle is the surprising lack of gas in $\mathrm{dSph}$ galaxies, where (with the exception of Sculptor; Bouchard, Carignan, \& Mashchenko 2003) only upper limits for neutral and ionized gas could be determined (Gallagher et al. 2003; Grebel et al. 2003, and references therein). Interestingly, the limits for neutral hydrogen lie well below the amounts expected from gas loss from the old red giants in the dSphs. Fornax, the only dSph galaxy with star formation as recent as $\sim 200 \mathrm{Myr}$ ago (Grebel \& Stetson 1999), surprisingly also appears to be devoid of gas. It is neither understood what caused the gas loss to begin with nor how it is sustained, especially in the distant, isolated dSphs like Cetus and Tucana.

When illustrating star formation histories via population boxes (e.g., Grebel 1997, 1999, 2000), it becomes quickly obvious that no two dwarf galaxies - not even lowmass dSphs - share the same star formation history. Each galaxy needs to be considered as an individual with regard to the time scales of its star formation and the degree of enrichment. A trend of increasing intermediate-age population fractions with increasing distance from the Milky Way among dSphs was first noted by van den Bergh (1994), 
who attributed it to the possible environmental impact of the Milky Way. Star-forming material might have been removed earlier on from the closer Galactic companions via ram-pressure or tidal stripping, supernova-driven winds or the high UV-flux from the proto-Milky Way. On the other hand, if environment is primarily responsible for gaspoor dSphs, then the existence of the isolated dSphs Cetus and Tucana is difficult to understand. Again, knowledge of the orbits of these galaxies would be very helpful.

We may then turn to the M31 dSph companions, which cover a similar range of distances as the Galactic ones. Interestingly, these dSphs do not show any clear correlation between star formation history and present-day distance to M31. Their lack of a pronounced red clump and of substantial number carbon stars shows a lack of any prominent intermediate-age population regardless of their distance (Harbeck et al. 2001, 2004, 2005). Going one step further, the comparison of the stellar populations in M31's dSphs and of M31's halo shows that the dSphs cannot have been primary building blocks of M31's halo since it was found to be dominated by intermediateage, comparatively metal-rich populations (Brown et al. 2003). (An old, metal-poor halo population, however, is present as well; Brown et al. 2004).

\subsection{Mean metallicities and the metallicity-luminosity relation}

Galaxies generally obey a metallicity-luminosity relation such that more luminous (and potentially more massive) galaxies are more metal-rich than faint galaxies with presumably more shallow potential wells. DIrrs and dSphs differ in their metallicityluminosity relations (e.g., Binggeli 1994). Originally this was discovered when comparing nebular $\mathrm{H}$ II region oxygen abundances of dIrrs with stellar metallicity estimates of gas-poor dwarfs such as dSphs. However, in making this comparison, very different populations are compared, and very different metallicity tracers are used. Nebular oxygen abundances cannot easily be translated into mean stellar metallicities and vice versa. Mean stellar metallicities usually rely on the measurement of iron or of an element that has been found to be an excellent tracer of iron in certain populations, such as the near-infrared Ca II triplet (Armandroff \& Da Costa 1991; Rutledge, Hesser, \& Stetson 1997; Cole et al. 2004). These mean stellar metallicities are usually quoted as $[\mathrm{Fe} / \mathrm{H}]$ or $[\mathrm{Me} / \mathrm{H}]$ (to indicate that primarily metallicity as opposed to solely $F e$ is meant).

In order to avoid the uncertain $\mathrm{O}$ to Fe conversion, Richer, McCall, \& Stasinska (1998) published a metallicity-luminosity relation based entirely on nebular O measurements: H II region abundances in dIrrs and planetary nebula (PN) abundances in dEs and dSphs. The offset between the resulting relations remained; however, one still compares different populations with each other. H II region abundances trace the presentday abundances of the most recently formed population of stars. PNe trace primarily intermediate-age populations with ages of at least several $100 \mathrm{Myr}$ if not several Gyr. Furthermore, PNe have only been detected in two dSphs to date (Fornax and Sagittarius). Obviously one cannot expect to measure $\mathrm{H}$ II regions in the gas-deficient dSphs.

In order to compare not only mean stellar metallicities in dIrrs and dSphs, but also the metallicities of the same populations (i.e., of stars of similar age), we instead concentrated on old Population II giants, which as mentioned earlier have been detected 
in all LG dwarf galaxies. We used (1) old red giants in dSphs and in the outskirts of dIrrs (where old populations dominate), (2) spectroscopic abundances wherever available (from own Keck LRIS measurements and literature data from studies conducted at ESO, NOAO, and Keck), and (3) photometric abundances elsewhere (from comparison with globular cluster fiducials applied to our own deep HST data from a WFPC2 snapshot survey and archival or literature data). While the degree of homogeneity of the resulting data set is not ideal, it is the best currently available one and entirely based on wellcalibrated empirical indicators.

The resulting metallicity-luminosity relationship shows that even when confined to old populations, there is a considerable offset between dSphs and dIrrs (Grebel et al. 2003). At the same galaxy luminosity, the old populations of dSphs are more metalrich than those of dIrrs. This indicates that in contrast to dIrrs, dSphs must have experienced fairly rapid early enrichment. Together with various other factors, these evolutionary differences make normal dIrrs unlikely progenitors of dSphs (see also Binggeli 1994). DIrr/dSph transition-type galaxies, on the other hand, seem fairly plausible progenitors as explained in more detail in Grebel et al. (2003).

\subsection{Detailed abundance ratios}

Mean spectroscopic metallicities of individual stars provide crucial constraints on otherwise photometrically derived star formation histories and allow one to break the agemetallicity degeneracy - one of the prime areas of study with the new large telescopes.

Another very important area for large telescopes is high-resolution spectroscopy to measure individual abundance ratios. In particular, the determination of $\mathrm{Fe}, \alpha-, \mathrm{r}_{-}$, and s-process element abundance ratios makes it possible to measure the modes and rates of star formation spectroscopically: the relative contribution of supernovae of Type II vs. Ia, and that of AGB stars, at different times during the evolution of the target galaxy. Importantly, this research permits one to compare the abundance ratios of different types of dwarf galaxies with those measured in various Galactic components and to constrain the building block scenario from the chemical point of view.

The $[\alpha / \mathrm{Fe}]$ ratios in dSphs (and dIrrs) at a given $[\mathrm{Fe} / \mathrm{H}]$ are lower than those measured in the Galactic halo, indicating either low star formation rates in the dwarfs, loss of metals, or a larger contribution from supernovae of Type Ia. This is strong evidence against present-day dSphs as the dominant contributors to the build-up of the Galactic halo (Shetrone et al. 2001). For a discussion of the implications of recent results for nucleosynthesis and galaxy evolution, see also Venn et al. (2004). Extending these kinds of measurements and adding kinematic data as well is rapidly becoming one of the major research areas for the world's largest telescopes such as SALT, nicely complemented by ongoing and future space missions such as HST, JWST, and Gaia.

\section{ACKNOWLEDGMENTS}

I would like to thank Joanna Mikolajewska for organizing a very stimulating conference and for her patience while this contribution was finished. 


\section{REFERENCES}

1. T. E. Armandroff, and G. S. Da Costa, $A J, 101,1329-1337$

2. T. E. Armandroff, J. E. Davies, and G. H. Jacoby, AJ, 116, 2287-2296 (1998).

3. T. E. Armandroff, G. H. Jacoby, and J. E. Davies, AJ, 118, 1220-1229 (1999).

4. B. Binggeli, "Dwarf galaxies: a morphological overview", in Panchromatic View of Galaxies. Their Evolutionary Puzzle, edited by G. Hensler, C. Theis, \& J. S. Gallagher, Editions Frontiers, Gif-surYvette, 1994, pp. 173-191

5. A. Bouchard, C. Carignan, and S. Mashchenko, AJ, 126, 1295-1304 (2003).

6. T. M. Brown, H. C. Ferguson, E. Smith, R. A. Kimble, A. V. Sweigart, A. Renzini, R. M. Rich, and D. A. VandenBerg, ApJ, 592, L17-L20 (2003).

7. T. M. Brown, H. C. Ferguson, E. Smith, R. A. Kimble, A. V. Sweigart, A. Renzini, and R. M. Rich, $A J$, 127, 2738-2752 (2004).

8. C. Brüns, et al., $A \& A, 432,45-67$ (2005).

9. P. I. Choi, P. Guhathakurta, and K. V. Johnston, $A J, \mathbf{1 2 4}, 310-331$ (2002).

10. A. A. Cole, T. A. Smecker-Hane, E. Tolstoy, T. L. Bosler, and J. S. Gallagher, MNRAS, 347, 367-379 (2004).

11. A. Dekel, and J. Woo, MNRAS, 344, 1131-1144 (2003).

12. A. E. Dolphin, NewA, 2, 397-409 (1997).

13. A. M. N. Ferguson, M. J. Irwin, R. A. Ibata, G. F. Lewis, and N. Tanvir, AJ, 124, 1452-1463 (2002).

14. A. Ferrara, and E. Tolstoy, MNRAS, 313, 291-309 (2000).

15. K. Freeman, and J. Bland-Hawthorn, ARA\&A, 40, 487-537 (2002).

16. J. S. Gallagher, G. J. Madsen, R. J. Reynolds, E. K. Grebel, \& T. A. Smecker-Hane, ApJ, 588, 326-330 (2003).

17. C. Gallart, A. Aparicio, G. Bertelli, and C. Chiosi, AJ, 112, 1950-1968 (1996).

18. G. Gilmore, R. F. G. Wyse, and J. E. Norris, ApJ, 574, L39-L42 (2002).

19. E. K. Grebel, Reviews in Modern Astronomy, 10, 27-59 (1997)

20. E. K. Grebel, "Evolutionary Histories of Dwarf Galaxies in the Local Group", in The Stellar Content of the Local Group, edited by P. Whitelock \& R. Cannon, IAU Symp. 192, Astronomical Society of the Pacific, San Francisco, 1999, pp. 17-38

21. E. K. Grebel, "The Star Formation History of the Local Group", in Star formation from the small to the large scale, edited by F. Favata, A.A. Kaas, \& A. Wilson, 33rd ESLAB Symposium, ESA-SP 445, ESA, Noordwijk, 2000, pp. 87-98

22. E. K. Grebel, A\&SSS, 277, 231-239 (2001).

23. E. K. Grebel, "The Evolutionary History of Local Group Irregular Galaxies", in Origin and Evolution of the Elements, edited by A. McWilliam \& M. Rauch, Carnegie Observatories Astrophysics Series, Vol. 4, Cambridge University Press, Cambridge, 2004, pp. 237-257

24. E. K. Grebel, and P. Guhathakurta, ApJ, 511, L101-L105 (1999).

25. E. K. Grebel, and P. B. Stetson, "Ground-based and WFPC2 Imaging of Fornax: Spatial Variations in Star Formation History", in The Stellar Content of the Local Group, edited by P. Whitelock \& R. Cannon, IAU Symp. 192, Astronomical Society of the Pacific, San Francisco, 1999, pp. 165-169

26. E. K. Grebel, and J. S. Gallagher, ApJ, 610, L89-L92 (2004).

27. E. K. Grebel, J. S. Gallagher, and D. Harbeck, AJ, 125, 1926-1939 (2003).

28. C. J. Grillmair, et al., $A J, \mathbf{1 1 5}, 144-151$ (1998).

29. D. Harbeck, et al., $A J, \mathbf{1 2 2}, 3092-3105$ (2001).

30. D. Harbeck, J. S. Gallagher, and E. K. Grebel, AJ, 127, 2711-2722 (2004).

31. D. Harbeck, J. S. Gallagher, E. K. Grebel, A. Koch, and D. B. Zucker, ApJ, in press (2005).

32. J. A. Holtzman, et al., AJ, 113, 656-668 (1997).

33. D. Hunter, PASP, 109, 937-950 (1997).

34. R. Ibata, G. Gilmore, and M. J. Irwin, Nature, 370, 194 (1994).

35. R. Ibata, M. Irwin, G. Lewis, A. M. N. Ferguson, and N. Tanvir, Nature, 412, 49-52 (2001).

36. C. Ikuta, and N. Arimoto, $A \& A, 391,55-65$ (2002).

37. H. Jerjen, B. Binggeli, and K. C. Freeman, AJ, 119, 593-608 (2000).

38. I. D. Karachentsev, and V. E. Karachentseva, A\&A, 341, 355-356 (1999).

39. I. D. Karachentsev, V. E. Karachentseva, A. A. Suchkov, and E. K. Grebel, A\&AS, 145, 415-423 (2000). 
40. I. D. Karachentsev, et al., $A \& A, \mathbf{3 8 9}, 812-824$ (2002a).

41. A. Klypin, A. V. Kravtsov, O. Valenzuela, and F. Prada, ApJ, 522, 82-92 (1999).

42. T. Lejeune, and J. Fernandes, Observed HR Diagrams and Stellar Evolution, edited by T. Lejeune \& J. Fernandes, ASP Conf. Proc., Vol. 274, Astronomical Society of the Pacific, San Francisco, 2002

43. L. Mayer, F. Governato, M. Colpi, B. Moore, T. Quinn, J. Wadsley, J. Stadel, and G. Lake, ApJ, 547, L123-L127 (2001).

44. N. F. Martin, N. F., R. A. Ibata, M. Bellazzini, M. J. Irwin, G. F. Lewis, and W. Dehnen, MNRAS, 348, 12-23 (2004a).

45. N. F. Martin, N. F., R. A. Ibata, B. C. Conn, G. F. Lewis, M. Bellazzini, M. J. Irwin, and A. W. McConnachie, MNRAS, 355, L33-L37, (2004b).

46. C. Mastropietro, B. Moore, L. Mayer, J. Wadsley, and J. Stadel, MNRAS, submitted (astro-ph/0412312), (2005).

47. M. Mateo, $A R A \& A, 36,435-506$ (1998).

48. Y. Momany, S. R. Zaggia, P. Bonifacio, G. Piotto, F. De Angeli, L. R. Bedin, and G. Carraro, $A \& A$, 421, L29-L32 (2004).

49. B. Moore, S. Ghigna, F. Governato, G. Lake, T. Quinn, J. Stadel, and P. Tozzi, ApJ, 524, L19-L22 (1999).

50. H. L. Morrison, P. Harding, D. Hurley-Keller, and G. Jacoby, ApJ, 596, L183-L186 (2003).

51. H. J. Newberg, et al., ApJ, 569, 245-274 (2002).

52. M. Odenkirchen, et al., ApJ, 548, L165-L169 (2001).

53. M. Odenkirchen, et al., AJ, 126, 2385-2407 (2003).

54. C. Palma, S. R. Majewski, M. H. Siegel, R. J. Patterson, J. C. Ostheimer, and R. Link, AJ, 125, 1352-1372 (2003).

55. S. Pedraz, J. Gorgas, N. Cardiel, P. Sánchez-Blázquez, and R. Guzmán, MNRAS, 322, L59-L63 (2002).

56. M. E. Putman, et al., Nature, 394, 752-754 (1998).

57. M. Richer, M. L. McCall, \& G. Stasinska, A\&A, 340, 67-76 (1998).

58. H. J. Rocha-Pinto, S. R. Majewski, M. F. Skrutskie, J. D. Crane, and R. J. Patterson, ApJ, 615, 732737 (2004).

59. G. A. Rutledge, J. E. Hesser, and P. B. Stetson, PASP, 109, 907-919 (1997).

60. J. L. Sérsic, Atlas de Galaxias Australes, Obs. Astron. de Córdoba, Córdoba, 1968.

61. M. D. Shetrone, P. Côté, and W. L. W. Sargent, ApJ, 548, 592-608 (2001).

62. T. A. Smecker-Hane, P. B. Stetson, J. E. Hesser, and M. D. Lehnert, AJ, 108, 507-513 (1994)

63. H. Susa, and M. Umemura, ApJ, 610, L5-L8 (2004).

64. K. Tassis, T. Abel, G. L. Bryan, and M. L. Norman, ApJ, 587, 13-24 (2003).

65. E. Tolstoy, and A. Saha, ApJ, 462, 672-683 (1996).

66. M. Tosi, L. Greggio, G. Marconi, and P. Focardi, AJ, 102, 951-974 (1991).

67. S. van den Bergh, ApJ, 428, 617-619 (1999).

68. S. van den Bergh, A\&ARv, 9, 273-318 (1999).

69. S. van den Bergh, The Galaxies of the Local Group, Cambridge Astrophysics Series Vol. 35, Cambridge University Press, Cambridge, 2000.

70. R. P. van der Marel, AJ, 122, 1827-1843 (2001).

71. F. van Leeuwen, J. D. Hughes, and G. Piotto, $\omega$ Centauri: A Unique Window into Astrophysics, edited by F. van Leeuwen, J. D. Hughes, \& G. Piotto, ASP Conf. Ser. 265, Astronomical Society of the Pacific, San Francisco, 2002.

72. K. A. Venn, M. Irwin, M. D. Shetrone, C. A. Tout, V. Hill, and E. Tolstoy, AJ, 128, 1177-1195 (2004).

73. M. I. Wilkinson, J. T. Kleyna, N. W. Evans, G. F. Gilmore, M. J. Irwin, and E. K. Grebel, ApJ, 611, L21-L24 (2004).

74. A. B. Whiting, G. K. T. Hau, and M. Irwin, AJ, 118, 2767-2774 (1999).

75. A. B. Whiting, G. K. T. Hau, and M. Irwin, ApJS, 141, 123-146 (2002).

76. R. F. G. Wyse, G. Gilmore, M. L. Houdashelt, S. Feltzing, L. Hebb, J. S. Gallagher, and T. A. Smecker-Hane, NewA, 7, 395-433 (2002).

77. B. Yanny, et al., ApJ, 588, 824-841 (2003).

78. D. Zaritsky, J. Harris, E. K. Grebel, and I. B. Thompson, ApJ, 534, L53-L56 (2000).

79. Zucker, D. B., et al., ApJ, 612, L121-L124 (2004a).

80. Zucker, D. B., et al., ApJ, 612, L117-L120 (2004b). 